\title{
Observation of Charge Generation via Photoinduced Stark Effect in Mixed- cation Lead Bromide Perovskite Thin Films
}

\author{
Nhu L. Tran ${ }^{1}$, Madeline H. Elkins ${ }^{1}$, David P. McMeekin ${ }^{2}$, Henry J. Snaith ${ }^{2}$, Gregory D. Scholes ${ }^{1}$ \\ 1 Department of Chemistry, Princeton University, Princeton, New Jersey 08544 United States \\ 2 Clarendon Laboratory, University of Oxford, Parks Road, Oxford OX1 3PU, UK
}

\section{Supplementary Methods}

\section{Sample preparation}

Substrate Cleaning: A glass slide was used as substrate. These glass substrates were sequentially cleaned with a $2 \%$ solution of Hellmanex detergent, then sonicated in acetone bath for 5 minutes, then sonicated in isopropyl alcohol (IPA) bath for 5 minutes, then the substrates were dried with a $\mathrm{N}_{2}$ spray gun. The glass slides were then cleaned for 10 minutes using an oxygen plasma.

$\mathrm{FA}_{\mathbf{0 . 8 3}} \mathrm{Cs}_{\mathbf{0 . 1 7}} \mathrm{PbBr}_{3}$ perovskite layer preparation: The $\mathrm{FA}_{0.83} \mathrm{Cs}_{0.17} \mathrm{PbBr}_{3}$ perovskite was prepared using an adaptation procedure proposed by F. Huang et al. [1]. The perovskite films were prepared by dissolving the precursor salts in anhydrous N,N-dimethylformamide (DMF) and dimethyl sulfoxide (DMSO) mixture (4:1 volume ratio) to obtain a stoichiometric solution with the desired $\mathrm{FA}_{0.83} \mathrm{Cs}_{0.17} \mathrm{PbBr}_{3}$ composition. The precursor solution was prepared using the following precursor salts: formamidinium bromide (FABr) (dyesol), ceasium bromide ( $\mathrm{CsBr})$ (99.9\%, Alfa Aesar), lead bromide $\left(\mathrm{PbBr}_{2}\right)(98 \%$, Alfa Aesar). The perovskite precursor solution was spin coated from a $0.5 \mathrm{M}$ solution at $4000 \mathrm{rpm}$ for $30 \mathrm{~s}$. A $\mathrm{N}_{2}$ gas was used to quench the growth of the perovskite. The gas flow was applied $15 \mathrm{~s}$ after the start of the spin coating, and sustained for $10 \mathrm{~s}$. The perovskite films were immediately placed on a preheated hot plate set to $100^{\circ} \mathrm{C}$ and annealed in a $\mathrm{N}_{2}$ atmosphere for 20 minutes. 
PMMA layer preparation: A $20 \mathrm{mg} / \mathrm{ml}$ solution of polymethyl methacrylate (PMMA) in anhydrous chlorobenzene (CB) was prepared. This solution was spin coated in a $\mathrm{N}_{2}$ glovebox at $2000 \mathrm{rpm}$ with a $1000 \mathrm{rpm}$ acceleration.

\section{BBTA and 2DES measurements:}

The output (800 nm at $5 \mathrm{kHz}$ with 100 -fs pulse duration) of a commercial Ti:Sapphire laser (Spectra Physics Spitfire Ace) is sent to a non-collinear optical parametric amplifier (NOPA) to generate broadband light centered at $540 \mathrm{~nm}(555 \mathrm{THz})$. The NOPA light is then compressed to 18-20 fs full-width at half-maximum (fwhm) for BBTA and 14-15 fs fwhm for 2DES as verified by polarization-gated and transient grating frequency-resolved optical gating (PG-FROG and TGFROG) respectively (see figures $\mathbf{S} 1$ and $\mathbf{S 2}$ ). The pulse compression is achieved by means of grating and prism compressors. At this point, the compressed pulse is directed to either the BBTA or 2DES setups.

BBTA: A wedged beam splitter passes $99 \%$ of the light intensity of the compressed pulse to be used as the pump and reflects the remaining $1 \%$ to be used as the probe and reference beams. The reference beam is necessary for balanced detection (for a detailed description, see [2]). The pumpprobe delay is scanned from $-0.2 \mathrm{ps}$ to $2 \mathrm{ps}$ in 4 -fs step size. The pump energy used is $0.5 \mathrm{~nJ}$ per pulse. 

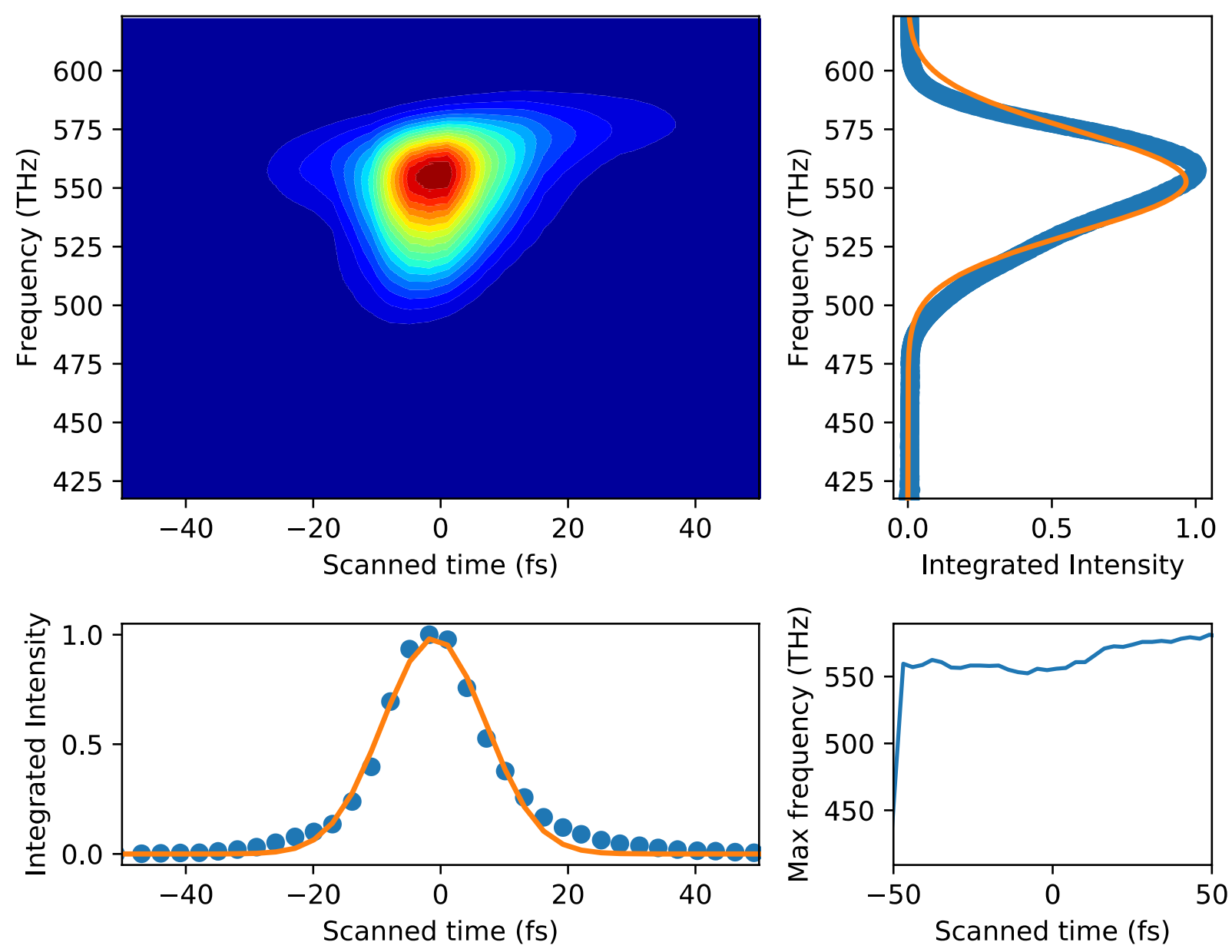

Figure S1: PG-FROG map of pulses used for BBTA measurements. Pulse duration: 19 fs.

2DES: The compressed pulse passes through a diffractive optic, splitting into four identical and phase-stable beams. Three of the four pulses then become the two pump pulses and probe pulse as described in the text. The fourth pulse is attenuated by a factor of $10^{4}$ to minimize interaction with the sample and serves as a reference in spectral interferometry with the third-order signal. The relative delays between pulses are controlled by translating the pairs of fused silica wedges inserted in the beam paths. The delay between the two pump pulses, $T_{1}$, is scanned between $-80 \mathrm{fs}$ and +80 fs in 0.2 -fs step size at each $T_{2}$ time. The population $T_{2}$ time is scanned from 0 to $452 \mathrm{fs}$ in 4 -fs step size. The pump energy used is also $0.5 \mathrm{~nJ}$ per pulse. For further information on the 2DES experimental setup, see [3]. 

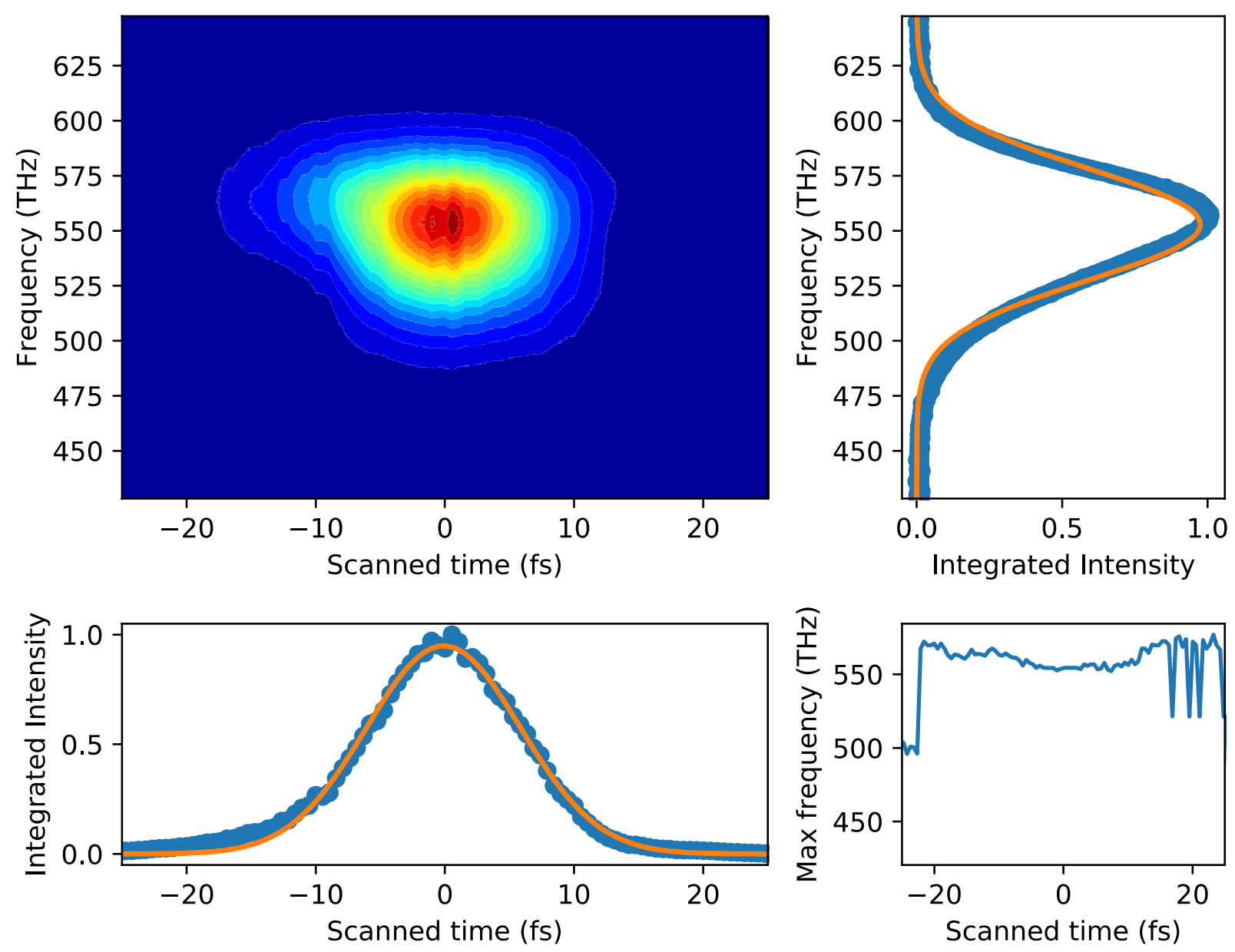

Figure S2: TG-FROG map of pulses used for 2DES measurements. Pulse duration: $14 \mathrm{fs}$.

\section{References}

1. Huang, F. et al. Gas-assisted preparation of lead iodide perovskite films consisting of a monolayer of single crystalline grains for high efficiency planar solar cells. Nano Energy (2014). doi:10.1016/j.nanoen.2014.08.015

2. McClure, S. D., Turner, D. B., Arpin, P. C., Mirkovic, T. \& Scholes, G. D. Coherent oscillations in the PC577 cryptophyte antenna occur in the excited electronic state. $J$. Phys. Chem. B (2014). doi:10.1021/jp411924c

3. Anna, J. M., Song Y., Dinshaw R. \& Scholes, G. D. Two-dimensional electronic spectroscopy for mapping molecular photophysics. Pure Appl. Chem. (2013). 
http://dx.doi.org/10.1351/PAC-CON-12-10-21 